\title{
Diabète
}

\section{et maladies parondontales}

\section{Diabetes and periodontal diseases.}

\section{MOTS-CLEFS :}

- Maladies parodontales, parodontites, diabète, inflammation

\section{KEYWORDS:}

- Periodontal diseases, periodontitis, diabetes, inflammation.
AOS 2014;267:27-34

DOI: $10.1051 /$ aos/2014105

(C) EDP Sciences 2014

\section{Résumé}

Le diabète et les maladies parodontales sont deux pathologies chroniques dont la prévalence mondiale est élevée et qui entretiennent des relations cliniques et biologiques complexes. L'augmentation de l'incidence et de la sévérité des parodontites chez les diabétiques est prouvée par de nombreuses études épidémiologiques. Les spécificités du traitement parodontal chez le diabétique sont liées à l'immunodépression qui engendre des retards de cicatrisation mais son efficacité est démontrée. Cependant les mécanismes pathogéniques sont encore loin d'être parfaitement élucidés. Depuis quelques années, la relation inverse entre parodontites et diabète a été l'objet de nombreuses recherches.

De récentes revues systématiques indiquent que les parodontites augmentent l'incidence, le contrôle et les complications du diabète et que le traitement parodontal classique (détartrage-surfaçage) diminue le taux d'hémoglobine glyquée de 0,4 \%. Là encore, les mécanismes biologiques sous-jacents restent à démontrer même si l'inflammation et le stress oxydant semblent impliqués.
Abstract

Diabetes and periodontal diseases are two chronic diseases with a high global prevalence and with complex clinical and biological relationships. Many epidemiological studies have shown an increased incidence and severity of periodontitis in diabetics. Immunosuppression in diabetics explains delory healing and specificity of periodontal therapy in those patients, but effectiveness of the treatment has been demonstrated. The pathogenic mechanisms are still far from being fully elucidated. In recent years, the inverse relationship between periodontitis and diabetes has been the subject of many researches. Recent systematic reviews indicate that periodontitis is responsible for increasing the incidence, control and complications of diabetes and that conventional periodontal treatment (scaling and root planning) reduces glycated haemoglobin of $0.4 \%$. The biological mechanisms implicated remain to be analysed however it appears that inflammation and oxidative stress are involved.

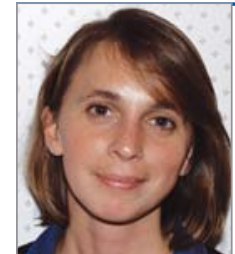

- Cécile Dagorne, Ancien Interne en Odontologie, European Postgraduate in Periodontology, Periodontics and Implant Dentistry, Département de parodontologie, Hôpital Rothschild, APHP, Paris, France

Hélène Rangé, AHU, Département de parodontologie, Hôpital Rothschild, APHP, Paris, France. UFR d'odontologie Garancière, Université Paris Diderot, Paris, France. INSERM U1148, hôpital Bichat, APHP, Paris, France 


\section{INTRODUCTION}

Le diabète est une maladie métabolique liée à une dysfonction de la régulation de la glycémie menant à une hyperglycémie chronique. Certains symptômes sont caractéristiques du diabète : polydipsie, polyurie, polyphagie, amaigrissement. Les complications du diabète correspondent à des lésions vasculaires microscopiques (rétine, rein) et macroscopiques (complications cardiovasculaires). Le terme « diabète » vient du grec et signifie " passer au travers " puisqu'à l'époque d'Hippocrate, les médecins avaient observés que «les malades étaient frappés d'une soif continuelle, et qu'ils semblaient uriner aussitôt ce qu'ils venaient de boire, comme s'ils étaient traversés par l'eau sans pouvoir la retenir ». Aujourd'hui, le diagnostic du diabète repose principalement sur la mesure de la glycémie (tableau 1). Toutefois, selon les mécanismes pathogéniques à l'origine de l'hyperglycémie, différentes formes de diabète sont distinguées : diabète de type 1 , de type 2 , gestationnel, essentiellement. Le diabète de type 1 , autrefois appelé diabète insulino-dépendant (ou encore diabète juvénile), est la forme la plus fréquente chez l'enfant, mais peut survenir à tous les âges et souvent de façon brutale accompagné de troubles de la vision et d'une fatigue inhabituelle. Il s'apparente à une maladie auto-immune où les cellules $\beta$ des îlots de Langerhans du pancréas (synthétisant l'insuline) sont détruites par le système immunitaire. L'insuffisance de sécrétion d'insuline dans le sang rend donc le patient diabétique de type 1 insulinodépendant. Le risque de transmission génétique de ce type de diabète est faible (autour de $5 \%$ ), les autres causes sont mal connues ce qui le rend difficilement évitable. En revanche, le diabète de type 2, qui repose sur une résistance des tissus cibles (foie, muscles) à l'action de l'insuline présente une composante génétique forte puisque le risque d'être diabétique de type 2 est de $30 \%$ quand un des parent est atteint et de $50 \%$ quand les deux parents le sont. De plus, près de $50 \%$ des diabétiques de type 2 sont obèses (indice de masse corporelle $\geq 30 \mathrm{~kg} / \mathrm{m}^{2}$ ) et $80 \%$ présentent un surpoids (IMC $\geq 25 \mathrm{~kg} / \mathrm{m}^{2}$ ). La pression génétique et environnementale est forte pour ce type de diabète qui peut donc être prévenu. Il représente près de $90 \%$ de l'ensemble des cas de diabète, il est généralement asymptomatique durant de longues années. Il faut souligner que son dépistage est simple et repose sur l'examen biologique de la glycémie facilement accessible au chirurgien dentiste en cas de signes d'alarme (infections récidivantes, difficultés de cicatrisation associées au contexte médical). Le diabète de type 2 est le plus souvent non insulinodépendant, mais un traitement par insuline peut être nécessaire pour la maîtrise de l'équilibre glycémique, d'où l'évolution actuelle de la terminologie. Le diabète de type 2 est souvent associé à d'autres facteurs de risque cardiovasculaire, comme l'hypertension artérielle, le surpoids et l'obésité, la dyslipidémie et le syndrome métabolique. Son incidence augmente de manière spectaculaire, en conséquence des modifications du mode de vie (sédentarité, alimentation hypercalorique) non seulement chez les adultes mais aussi chez les enfants.

Les complications du diabète sont multiples, essentiellement nerveuses et vasculaires. Il faut distinguer les atteintes des gros vaisseaux dues à l'athérosclérose (infarctus du myocarde, accidents vasculaires cérébraux, artériopathie oblitérante des membres inférieurs) des atteintes microangiopathiques caractéristiques des diabétiques : rétinopathies, néphropathies. Les neuropathies parfois douloureuses peuvent alourdir les complications vasculaires notamment dans les situations d'artériopathie des membres inférieurs où la diminution de la sensibilité douloureuse peut entraîner un retard de prise en charge de plaies du pied (mal perforant plantaire).

Enfin, sur le plan biologique, le diabète est caractérisé par une dysfonction immunitaire et un état inflammatoire de bas grade qui sont source d'infection. Les maladies parodontales sont depuis longtemps reconnues comme une des complications du diabète et les preuves épidémiologiques et biologiques de cette relation sont nombreuses. Plus récemment, les études cliniques ont montré un impact des parodontites sur le contrôle de la glycémie, l'incidence et les

Tableau 1 : Valeurs de la glycémie et signification clinique (d'après l'American Diabetes Association en 2004)

\begin{tabular}{|c|c|}
\hline Valeurs de la glycémie & Signification clinique \\
\hline De 0,8 et $1,10 \mathrm{~g} / \mathrm{L}$ (à jeun) & Sujet non diabétique \\
\hline De 1,10 à 1,25 (à jeun) & $\begin{array}{l}\text { Intolérance au } \\
\text { glucose - hyperglycémie }\end{array}$ \\
\hline I Supérieure ou égale à 1,26 g/L (à jeun) & Sujet diabétique \\
\hline $\begin{array}{l}\text { I Supérieure ou égale à } 2 \mathrm{~g} / \mathrm{L} \text { (associée à la triade } \\
\text { polydipsie, polyurie, amaigrissement) }\end{array}$ & Sujet diabétique \\
\hline $\begin{array}{l}\text { I Supérieure ou égale à } 2 \mathrm{~g} / \mathrm{L} \text { ( } 2 \text { heures après l'ingestion } \\
\text { de } 75 \mathrm{~g} \text { de glucose par voie orale) }\end{array}$ & Sujet diabétique \\
\hline
\end{tabular}


complications du diabète. L'objectif de cet article est de faire la synthèse des connaissances actuelles sur cette relation bidirectionnelle entre le diabète et les maladies parodontales.

\section{NOTIONS D'ÉPIDÉMIOLOGIE SUR LE DIABÈTE ET LES MALADIES PARODONTALES}

\section{Prévalence des maladies parodontales et du diabète}

La parodontite chronique est la cause la plus fréquente d'avulsion chez les plus de 40 ans. La prévalence de la parodontite chronique chez les adultes varie de 20 à $50 \%$ selon les études, la plaçant en tête des maladies inflammatoires chroniques dans le monde [1]. Dans une revue de la littérature menée par Mealey et al. en 2007 [2], il est rapporté que le diabète de type 1 est une des pathologies chroniques les plus fréquentes de l'enfance. De plus, les pays scandinaves montrent les plus fortes incidences de diabète de type 1 avec plus de 30 cas/an/100 000 habitants, suivis par l'Europe et les États-Unis (10-15 cas/an/100 000 habitants) puis par les pays asiatiques avec $0,5 \mathrm{cas} / \mathrm{an} / 100000 \mathrm{habi}-$ tants [2]. La prévalence mondiale du diabète de type 2 était de 150 millions en 2000 et de 220 millions en 2010. Selon l'OMS, en mars 2013, 347 millions de personnes étaient diabétiques dans le monde (type 1 et 2) et il est prévu que le diabète sera la septième cause de décès dans le monde avec 439 millions de personnes atteintes en 2030. La progression rapide et constante du nombre de malades (plus $54 \%$ en 20 ans), tant dans les pays développés que dans les pays en voie de développement, signe l'urgence d'une prise en charge pluridisciplinaire de ce problème majeur de santé publique. Aujourd'hui près de $10 \%$ de la population mondiale est diabétique.

\section{Association maladies parodontales et diabète : données épidémiologiques}

Il existe des preuves épidémiologiques fortes du rôle du diabète dans le développement et la sévérité des maladies parodontales. En France, une étude portant sur plus de 2000 sujets a montré que la perte d'attache chez les diabétiques de type 2 était significativement plus élevée (parodontite plus sévère) que chez les non diabétiques $(2,7 \mathrm{~mm}$ versus $2,5 \mathrm{~mm}, \mathrm{p}=0,001)$ [3]. La National Health and Nutrition Examination Survey, aux États-Unis entre 1999-2000, a donné une prévalence des parodontites chez les sujets diabétiques et non diabétiques de respectivement $12 \%$ et $6 \% \mathrm{chez}$ les Afro-Américains, $15 \%$ et $4 \%$ chez les Latino-Américains, $12 \%$ et $3 \%$ chez les Caucasiens [4]. Plus récemment, un rôle des parodontites sur l'incidence, le contrôle et les complications du diabète a été mis en évidence. Le rapport de consensus du Workshop 2013 commun à l'EFP et l'AAP traitant de «maladies parodontales et pathologies systémiques » [5] a montré les éléments suivants :

$\checkmark$ évidence forte de l'influence négative des parodontites sévères sur le niveau d'HbA1c chez les sujets avec ou sans diabète ;

$\checkmark$ relation directe entre la sévérité des parodontites et les complications du diabète de type 2 ;

$\checkmark$ évidence limitée de l'association entre maladies parodontales et complications du diabète chez des adultes atteints depuis longtemps de diabète de type 1 ;

$\checkmark$ l'évidence disponible à ce jour suggère un rôle dose-dépendant des parodontites sur le diabète : risque augmenté de développer un diabète chez les individus atteints de parodontite sévère comparativement à ceux ayant une parodontite modérée ou un terrain parodontal sain.

En 2013, dans une revue systématique épidémiologique Borgnakke et ses collaborateurs [6] ont confirmé qu'il existait à ce jour un nombre limité d'études à haut niveau de preuve (petits échantillons) attestant de manière significative des effets néfastes des parodontites sur le contrôle glycémique, les complications du diabète et le développement d'un diabète de type 2 ou gestationnel. À ce jour, la littérature scientifique suggère une influence négative des maladies parodontales sur le diabète, cependant des études longitudinales sont nécessaires à la confirmation de ces résultats.

\section{MÉCANISMES BIOLOGIQUES DU LIEN ENTRE LE DIABÈTE ET LES MALADIES PARODONTALES}

\section{Influence du diabète sur les maladies parodontales (fig.1)}

Les taux plus élevés de glucose dans le fluide gingival des patients diabétiques pourraient constituer un environnement favorable au développement d'une flore microbienne spécifique et plus pathogène. Cependant, les nombreuses études cliniques transversales sur la composition de la plaque sous-gingivale entre patients diabétiques et non diabétiques n'ont pas permis de mettre en évidence un impact du diabète sur le microbiote parodontal. Il n'existe pas non plus de lien entre le niveau de contrôle de la glycémie (HbAlc) chez les diabétiques et la composition de la plaque sous-gingivale [7]. 


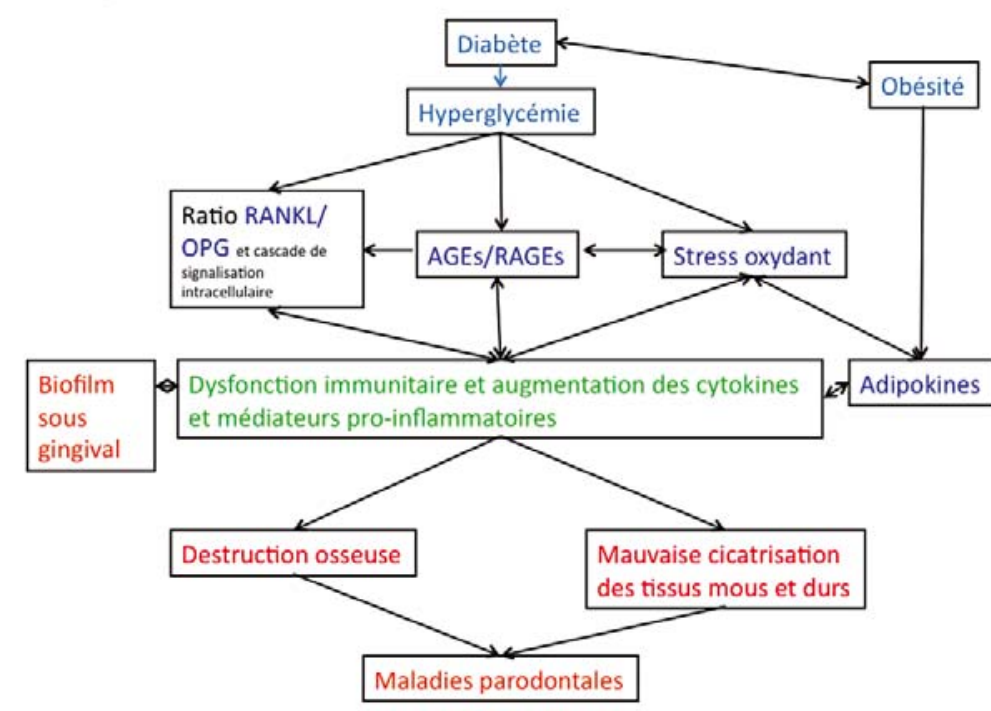

Figure 1 :

Mécanismes biologiques impliquant le diabète dans la pathogenèse des maladies parodontales (d'après Taylor et al 2013).

RANKL/OPG : Receptor Activator of Nuclear Factor-kappa B Ligand/Osteoprotegerin; AGEs/RAGEs : Advanced Glycation end-products/Receptor of Advanced Glycation End-products

\section{Rôle de la dysfonction immunitaire et de la réaction inflammatoire exacerbée}

De nombreuses études se sont intéressées à la dysfonction des polynucléaires neutrophiles chez le diabétique en lien avec la prévalence et la sévérité des maladies parodontales. Certaines études animales et cliniques semblent montrer que l'adhérence, le chimiotactisme (diminution de l'IL-8) et la phagocytose des neutrophiles tant périphériques que gingivaux sont altérés chez les patients diabétiques de type 2 [8]. De même, le rôle des monocytes dits " hyper répondants " ou «hyper inflammatoires » est soulevé dans la susceptibilité des patients diabétiques aux maladies parodontales. Les études de Salvi et de ses collaborateurs en 1997 [9] ont montré que les monocytes des patients diabétiques de type 1 produisaient plus de cytokines et médiateurs pro-inflammatoires tels que l'interleukine 1 bêta (IL-1 $\beta$ ), le tumor necrosis factor alpha (TNF $\alpha$ ) et la prostaglandine $\mathrm{E}_{2}\left(\mathrm{PGE}_{2}\right)$ en réponse à l'agression bactérienne (notamment au lipopolysaccharide ou LPS) que les monocytes de patients non diabétiques [9]. Plus récemment, un troisième type cellulaire a été impliqué dans la pathogenèse des maladies parodontales chez le diabétique : les lymphocytes T. En effet, les lymphocytes $\mathrm{T}$ auraient un rôle dans le dysfonctionnement de l'infiltrat macrophagique observé dans le tissu adipeux chez les patients obèses et ou diabétiques. Différentes sous-populations de lymphocytes $\mathrm{T}$ existent suivant l'environnement cytokinique pro ou anti-inflammatoire. Il apparait que les patients atteints de parodontite et de diabète de type 2 non équilibré auraient plus de lymphocytes Th 17 (sécrétant l'interleukine 17, pro-inflammatoire) et de $T$ régulateurs que les patients atteints de parodontite mais non diabétiques [10]. Enfin, l'implication des cytokines, adipokines et médiateurs pro-inflammatoires dans la pathogenèse des maladies parodontales chez les diabétiques fait aujourd'hui consensus. Dans le cas du diabète de type 1, les études de Salvi et collaborateurs sur le fluide gingival de patients diabétiques et non diabétiques atteints de parodontite de même sévérité ont montré des taux plus élevés d'IL-1 $\beta$, de $\mathrm{PGE}_{2}$ et de métallo-protéinases (MMPs) [9]. Pour le diabète de type 2 , les résultats des études cliniques, bien que controversés, semblent indiquer une augmentation des taux d'IL- $1 \beta$ et d'IL- 6 avec le diabète chez les patients atteints de parodontite chronique [7] ainsi qu'avec le taux d'hémoglobine glyquée ou HbAlc. D'autres médiateurs de l'inflammation pourraient participer à la pathogenèse des parodontites chez les diabétiques de type 2 tels l'interféron $\gamma$ (IFN $\gamma$ ), la macrophage inhibitory protein-2 (MIP-2), la monocyte chemotactic protein-1 (MCP-1) ou encore le TNFa. Cependant, seules des études animales ont apporté la preuve de l'implication de TNFa dans le développement des parodontites chez les rongeurs diabétiques de type 1 et 2 [11]. Les cytokines et médiateurs pro-inflammatoires ne sont pas les seuls mécanismes moléculaires impliqués dans la pathogenèse des maladies parodontales chez le sujet diabétique. Des études récentes ont montré que le ratio entre le ligand du récepteur activateur du facteur nucléaire kappa $\mathrm{B}$ et l'ostéoprotégérine (RANKL/OPG) était augmenté chez les diabétiques de type 2 atteints de parodontite chronique [12] et positivement associé au contrôle de la glycémie (fig. 2). Enfin, les adipokines (cytokines produites par le tissu adipeux) telles que la leptine, la résistine ou l'adiponectine, impliquées dans le lien entre l'obésité et les maladies parodontales [13] pourraient l'être également dans le diabète mais les études peu nombreuses restent controversées.

\section{Rôle de l'hyperglycémie sur les advanced glycation end-products et leurs récepteurs (AGEs/RAGEs), l'inflammation, le stress oxydant et le métabolisme osseux}

Si il y a carence ou mauvaise utilisation de l'insuline, le glucose ne peut pas pénétrer dans les cellules de stockage (muscle, tissu adipeux, foie). Lorsque les protéines et les lipides plasmatiques et membranaires sont exposés pendant un temps long à une haute concentration en glucose (hyperglycémie), elles subissent une modification structurelle non enzymatique et irréversible : la glycation. Ces protéines modifiées sont appelées advanced glycation end products (AGEs) et sont présentes sur les 


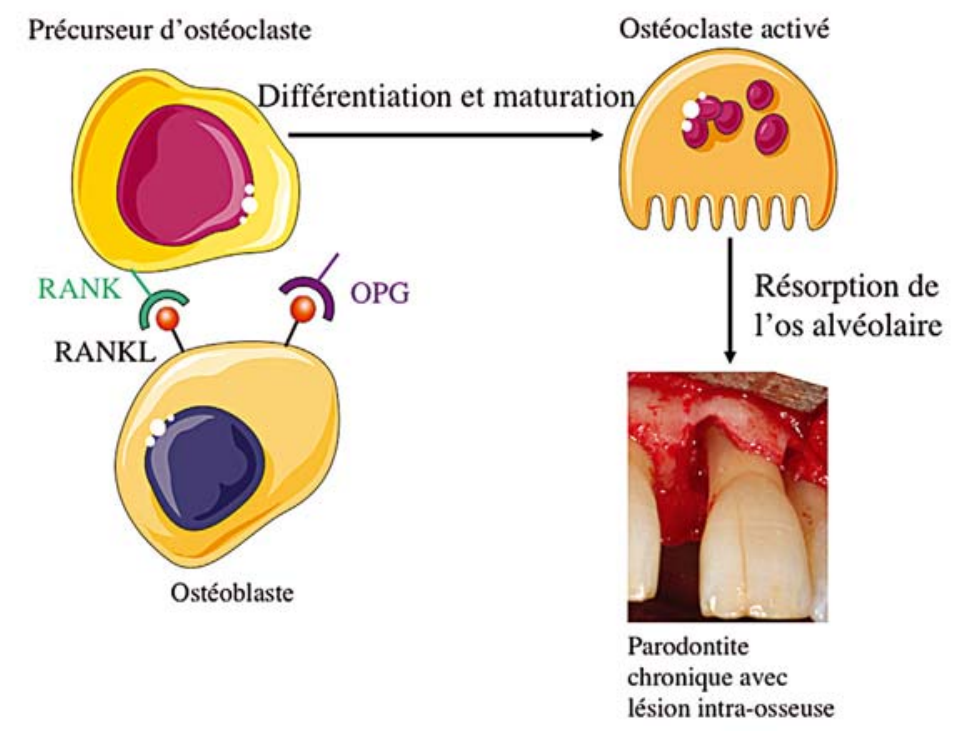

\begin{abstract}
$<$ Figure 2 :
Schéma synthétique du système RANKL/RANK/OPG. L'ostéoprotégérine $(\mathrm{OPG})$, sécrétée par les ostéoblastes, entre en compétition avec le ligand au récepteur-activateur du facteur nucléaire kappa B (RANKL) pour le récepteuractivateur du facteur nucléaire kappa $B$ (RANK) porté par les précurseurs d'ostéoclastes pour neutraliser leur différenciation et leur maturation en ostéoclastes matures. Un ratio RANKL/OPG élevé est en faveur de l'ostéoclastogenèse et donc de la résorption osseuse.
\end{abstract}

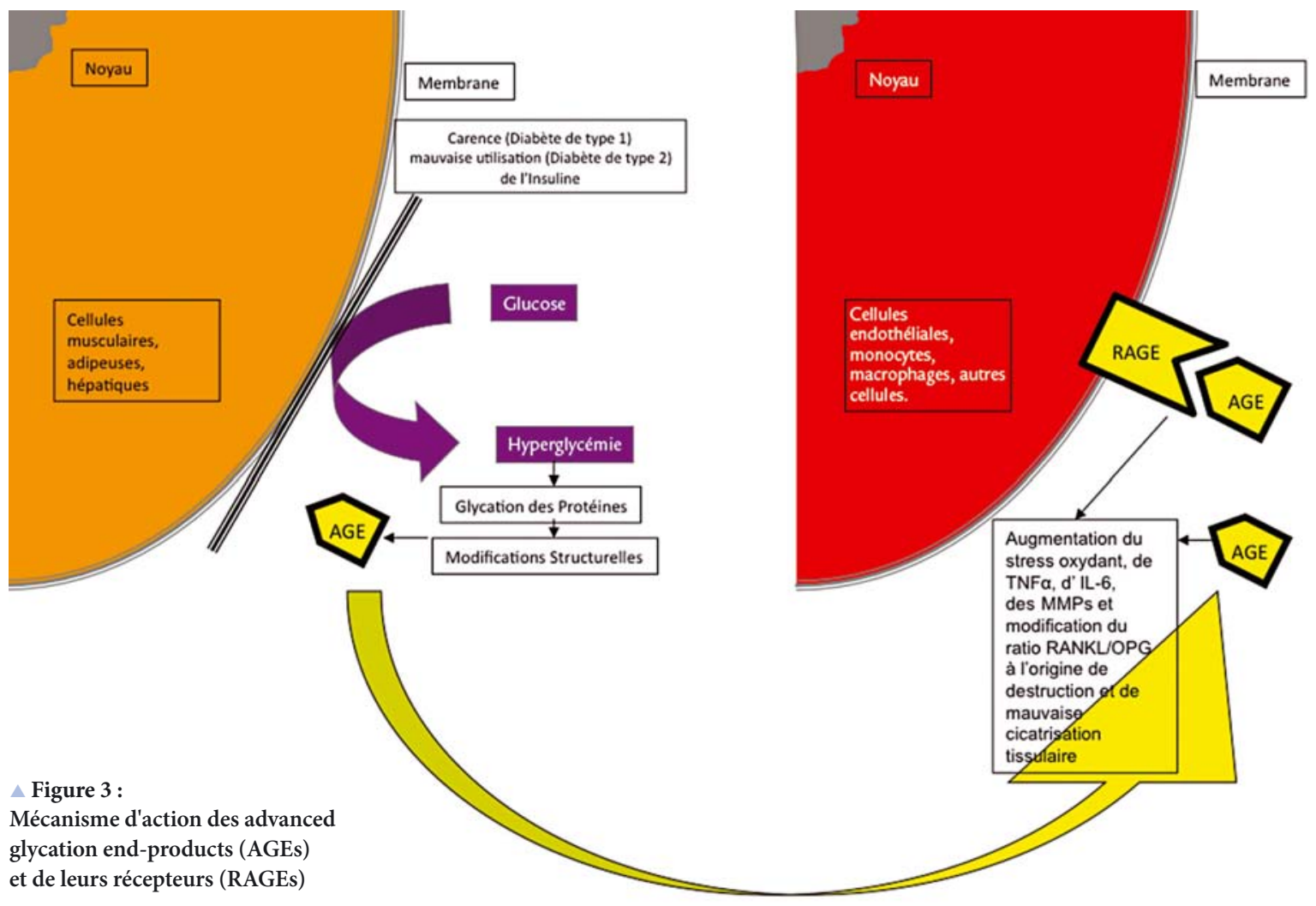

protéines plasmatiques, sur les protéines des parois vasculaires et aussi à la surface des globules rouges. Les AGEs ont un pouvoir chimiotactique sur les monocytes et les macrophages. Elles se lient spécifiquement à des récepteurs (immunoglobulines) appelés RAGEs situés à la surface de ces cellules. Les monocytes et macrophages ainsi activés produisent alors plus de radicaux libres oxygénés (stress oxydant) et de cytokines pro-inflammatoires conduisant ainsi à diverses complications du diabète dont les maladies parodontales [14, 15] (fig. 3). Une étude animale chez le rongeur diabétique a montré que l'apport de RAGEs solubles diminuait la production de TNF, d'IL-6 et de MMPs dans les tissus parodontaux et empêchait l'alvéolyse [16]. Les AGEs pourraient aussi intervenir dans la pathogenèse des maladies parodontales par la voie des toll-like receptors (TLR), à l'origine de la réponse inflammatoire induite par le LPS des bactéries.

L'hyperglycémie est également source de stress oxydant, avec une répercussion pro-inflammatoire, pour de nombreux types cellulaires (monocytes, fibroblastes, cellules épithéliales) et ce par divers mécanismes de signalisation intra-cellulaire comme la voie des MAP kinases, du facteur nucléaire kappa $B(N F-\kappa B)$ et de l'inflammasome NALP3. Les radicaux libres oxygénés (Reactive Oxygen Species) ont également un effet sur le métabolisme osseux et pourraient donc intervenir par ce biais sur la pathogenèse des parodontites [7]. 
Enfin, l'hyperglycémie modulerait, directement et indirectement par le système AGEs/RAGEs, le ratio RANKL/OPG en faveur de l'ostéoclastogenèse et donc de l'aléolyse chez le diabétique.

\section{Influence des parodontites sur le diabète}

Bien que de nombreuses études épidémiologiques aient montré un effet des parodontites sur le taux d'HbAlc, l'équilibre et les complications du diabète, les études cliniques et animales l'ont encore rarement démontré.

\section{Rôle de la flore parodontopathogène}

Une étude d'intervention a montré qu'une souche de Porphyromonas gingivalis (fimbriae type II) pourrait influer sur le contrôle de la glycémie chez des patients diabétiques et atteints de parodontite chronique [7]. Cette souche de $P$. gingivalis induirait des taux plus élevés de cytokines pro-inflammatoires que les autres. Toutefois, à ce jour, les études ne sont pas assez nombreuses pour affirmer un rôle du microbiote parodontal sur l'équilibre de la glycémie.

\section{Rôle des facteurs inflammatoires}

Un état inflammatoire chronique de bas grade, à l'instar de ce qui est observé dans l'obésité, est maintenant considéré comme un environnement à risque de pré-diabète de type 1 et 2 . Il paraît plausible que les parodontites, en augmentant les taux in situ et systémiques de médiateurs pro-inflammatoires comme la protéine $C$ réactive (CRP), le TNFa et l'IL-6, favorisent le développement du diabète. De plus, les radicaux libres produits par les monocytes activés au cours des parodontites pourraient aussi favoriser l'inflammation et le pré-diabète [18].

\section{Diabète et traitement des maladies parodontales}

\section{Bénéfice du traitement parodontal chez le sujet diabétique}

En 2013, Engebretson et ses collaborateurs [19] ont effectué une méta-analyse dans le but d'étudier les effets du traitement parodontal chez le sujet diabétique. Les critères d'inclusion étaient les suivants :

$\checkmark$ essais randomisés contrôlés ;

Iraitement parodontal chez des sujets diabétiques ; groupe contrôle non traité ;

suivi sur 3 mois.

Ces critères n'ont été retrouvé que pour 9 publications, les résultats de la méta-analyse montraient que le traitement parodontal permettait une diminution significative de $0,36 \%$ (IC $95 \%-0,54 ;-0,19$ ) des niveaux d'HbA1c chez les sujets diabétiques traités comparativement au groupe contrôle $(p<0,0001)$. Les études sélectionnées ne concernaient que des sujets diabétiques de type 2 .

D'autres méta-analyses effectuées en 2010 donnaient les même résultats : diminution de l'HbAlc de $0,40 \%$ après traitement parodontal $[20,21]$. En 2008, Darré et ses collaborateurs ont également réalisé une méta-analyse, cette dernière montrait une diminution de l'HbAlc de 0,46\%. La diminution de l'HbAlc permet un meilleur contrôle du diabète et ainsi de limiter l'apparition de complications.

Les traitements parodontaux effectués dans ces études sont variés mais comprennent toujours des débridements mécaniques professionnels (détartrages et surfaçages radiculaires), avec ou sans antibiothérapie, associés à l'enseignement de l'hygiène orale [5].

Les résultats présentés demandent à être confirmés par des études multicentriques avec de plus grands échantillons de population. En ce qui concerne les sujets diabétiques de type 1 , nous ne disposons pas à ce jour de résultats avec un niveau de preuve suffisant.

\section{Gestion du diabète pendant le traitement parodontal}

\section{Pendant la thérapeutique initiale}

En 1998, Christgau et collaborateurs ont comparé les résultats du traitement de parodontites modérées à sévères (indice de plaque, inflammation, indice de saignement, profondeur de poche, niveau d'attache, microbiologie) entre un groupe de patients diabétiques (HbA1 c = 4,4-10,6\%, 7 diabétiques de type 1 et 13 diabétiques de type 2 ) et un groupe contrôle de patients non diabétiques $(n=20)$. Les résultats montraient, quatre mois après la thérapeutique non chirurgicale, une amélioration significative de tous les paramètres parodontaux sans différence entre les sujets diabétiques et les non diabétiques.

Les recommandations du dernier Workshop EFP/ AAP [5] préconisaient, chez les sujets diabétiques de type 2 , une prise en charge des maladies parodontales par un traitement parodontal classique : débridement mécanique professionnel et enseignement de l'hygiène orale (fig. 4 et 5). En effet, il n'y a pas, à ce jour, d'évidence de supériorité d'un traitement spécifique ou de l'adjonction d'une antibiothérapie. De plus, les sujets diabétiques doivent être informés de leur plus grand risque de développer une maladie parodontale et d'un moins bon contrôle des paramètres glycémiques en cas de parodontite non traitée. Ces sujets doivent recevoir chaque année un examen parodontal complet. Enfin, il est nécessaire d'adresser à un spécialiste (endocrinologue) les patients non diagnostiqués comme diabétiques mais présentant des signes de maladie parodontale associée à des signes de diabète de type 2 (polyurie, 
$\checkmark$ Tableau 2. Prise en charge des patients diabétiques avec ou sans maladie parodontale

\begin{tabular}{|c|c|c|}
\hline & Diabétiques diagnostiqués & Diabétiques non-diagnostiqués \\
\hline $\begin{array}{l}\text { Phase systémique } \\
\text { du traitement parodontal }\end{array}$ & $\begin{array}{l}\text { Vérifier les taux d'HbAtc et la bonne prise en } \\
\text { charge du patient pour son diabète. } \\
\text { Si nécessaire, contacter le médecin traitant. }\end{array}$ & $\begin{array}{l}\text { Maladie parodontale associée à des signes } \\
\text { de diabète : détection des taux d'HbAıc } \\
\text { et adresser le patient à un spécialiste. }\end{array}$ \\
\hline $\begin{array}{l}\text { Thérapeutique parodontale } \\
\text { initiale }\end{array}$ & \multicolumn{2}{|c|}{$\begin{array}{l}\text { Débridement mécanique professionnel et enseignement de l'hygiène orale. } \\
\text { Pas de preuve de l'intérêt de l'adjonction d'une antibiothérapie. }\end{array}$} \\
\hline $\begin{array}{l}\text { Thérapeutique parodontale } \\
\text { chirurgicale et/ou implantaire }\end{array}$ & \multicolumn{2}{|c|}{ Possible si $\mathrm{HbA} 1 \mathrm{c}<7 \%$. } \\
\hline & Diabétiques sans maladie parodontale & Diabétiques avec maladie parodontale \\
\hline Suivi des patients & $\begin{array}{l}\text { Réaliser un examen parodontal complet } \\
\text { chaque année. Informer le patient } \\
\text { des risques augmentés de développer } \\
\text { une maladie parodontale. }\end{array}$ & $\begin{array}{l}\text { Adapter la fréquence des séances de thérapeutique } \\
\text { parodontale de soutien. Informer le patient } \\
\text { du risque de diminution du contrôle glycémique } \\
\text { si la parodontite n'est pas traitée. }\end{array}$ \\
\hline
\end{tabular}

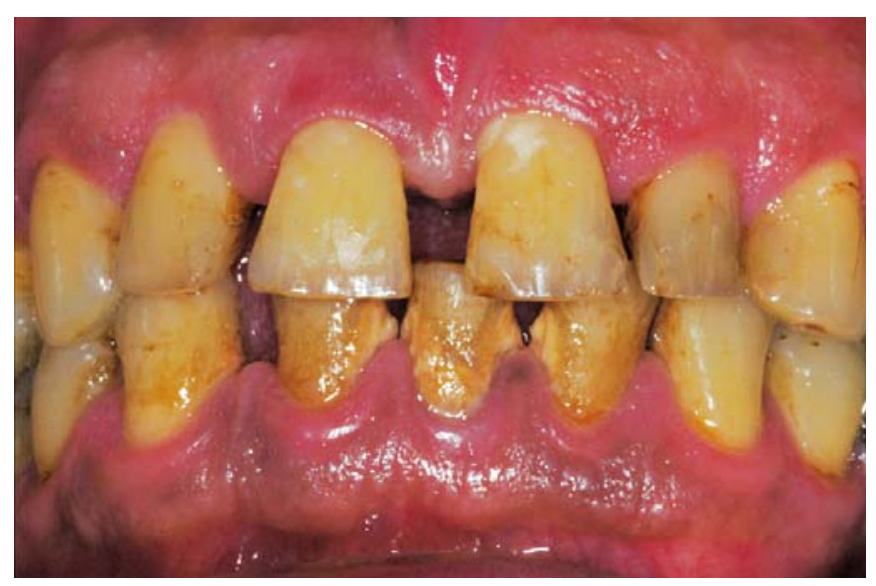

$\triangle$ Figure 4 :

Parodontite chronique sévère chez un patient diabétique de type 2. Situation clinique avant traitement parodontal.

polydipsie, polyphagie). Dans ce cas, la mesure du taux d'HbAlc, simple et rapide, peut être prescrite par le chirurgien dentiste en première intention.

Chez les sujets diabétiques de type 1, l'intérêt de la doxycycline parallèlement au traitement parodontal non chirurgical a été étudié en administration locale et systémique. En 2004, Martorelli de Lima et ses collaborateurs, ont réalisé une étude clinique, en bouche comparée, sur 22 défauts parodontaux résiduels ( $\geq 5 \mathrm{~mm}$ ) après la thérapeutique initiale, chez 11 sujets. Les résultats montraient, de manière significative $(\mathrm{p}<0,05)$, une plus grande diminution de la profondeur de poche et un plus grand gain d'attache dans le groupe test (détartrage-surfaçage radiculaire et gel de doxycycline en sous-gingival), comparativement au groupe contrôle (détartrage-surfaçage radiculaire + gel placebo en sous-gingival) 12 mois après le traitement.

En 2005, Llambés et ses collaborateurs [24] ont analysé les résultats du traitement parodontal non chirurgical chez 60 sujets diabétiques de type 1 . Le groupe test $(n=30)$ avait reçu, en plus du traitement parodontal mécanique initial, une administration systémique de

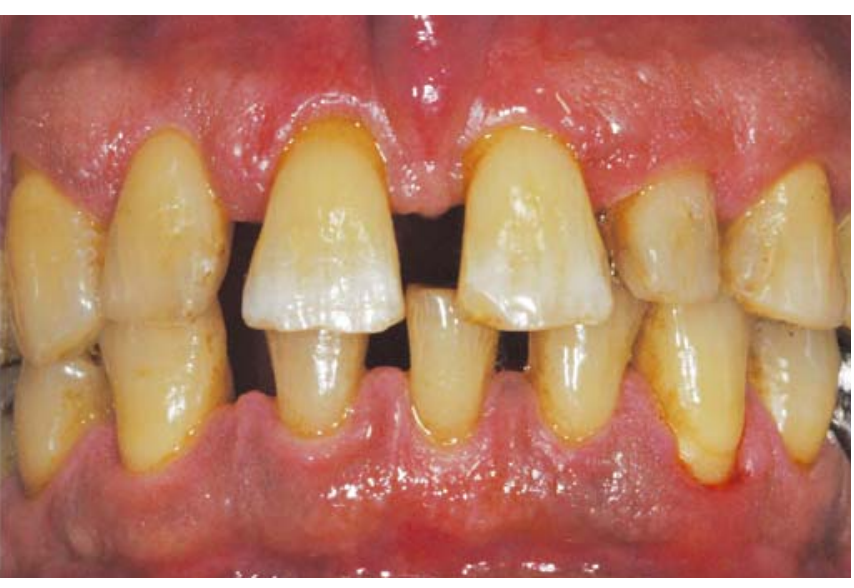

$\triangle$ Figure 5 :

Parodontite chronique sévère chez un patient diabétique de type 2 . Situation clinique 8 semaines après la thérapeutique initiale (enseignement à l'hygiène orale, détartrage et surfaçages radiculaires).

doxycycline pendant 15 jours et le groupe contrôle $(\mathrm{n}=30)$ n'avait pas reçu de doxycycline. 12 semaines après le traitement, la réduction des profondeurs de poche pour les poches profondes $(\geq 6 \mathrm{~mm})$ et du saignement au sondage était significativement supérieure dans le groupe test $(p=0,03)$. Cependant les résultats de ces deux études demandent à être confirmés par des études de plus haut niveau de preuve sur de plus grands échantillons de population.

\section{Cicatrisation et chirurgie parodontale ou implantaire}

Les taux sériques d'HbA1c reflètent le contrôle de la glycémie lors des trois derniers mois. Un diabète est dit équilibré lorsque le taux d'HbA1c est $<$ à $7 \%$. Chez les patients diabétiques devant recevoir un traitement parodontal chirurgical ou un traitement implantaire, il est donc nécessaire d'obtenir des taux d'HbA $1<$ à $7 \%$. Une communication entre le dentiste ou le parodontiste et le médecin traitant peut alors s'avérer indispensable. Des taux d'HbA1c trop élevés entraîneront des complications pour la cicatrisation 
postopératoire. Le tableau 2 résume les spécificités du traitement parodontal chez les patients diabétiques ainsi que les précautions à mettre en ouvre lors du suivi de ces patients en parodontologie.

\section{CONCLUSION}

Le diabète, avec le tabac, est un des deux facteurs de risque majeurs des maladies parodontales. Les mécanismes biologiques impliqués dans la pathogenèse des parodontites chez le patient diabétique sont de mieux en mieux connus et reposent sur la dysrégulation de médiateurs pro-inflammatoires comme l'IL-1 $\beta$, l'IL-6, le TNFa et de molécules clés du métabolisme osseux et de la cicatrisation tels que le système RANKL/OPG et AGEs/RAGEs. Les patients diabétiques sont et seront aussi de plus en plus fréquents au cabinet dentaire puisque près de $10 \%$ de la population mondiale est diabétique. L'influence croissante du surpoids et de l'obésité renforce cette prise de conscience sanitaire. Si les parodontites sont plus fréquentes et plus sévères chez les diabétiques, leur traitement ne diffère pas et les résultats sont bons [25]. Toutefois les relations qu'entretiennent le diabète et les maladies parodontales sont complexes et depuis quelques années les preuves s'accumulent également dans le sens inverse. Le cercle est vicieux. Les maladies parodontales déséquilibrent la glycémie, augmentent le risque de complication chez le diabétique et pourraient être un facteur de risque de diabète. Le traitement parodontal est bénéfique chez les diabétiques puisque les méta-analyses s'accordent sur une réduction d'environ $0,4 \%$ du taux d'hémoglobine glyquée (HbA1c), ce qui équivaut au bénéfice thérapeutique de l'administration d'un deuxième médicament anti-diabétique pour le patient. Il apparaît clair que les maladies parodontales doivent être prévenues et traitées efficacement, non seulement pour conserver une denture fonctionnelle à long terme mais aussi pour réduire le risque de pathologies métaboliques comme le diabète.

\section{Bibliographie}

[1] Eke P.I. et al. Prevalence of Periodontitis in adults in the United States : 2009 and 2010. J Dent Res (2012) 91:914-920.

[2] Mealey B.L. et al. Diabetes mellitus and periodontal disease. Periodontol 2000 (2007) $44: 127-153$.

[3] Mattout C, Bourgeois D, Bouchard P. Type 2 diabetes and periodontal indicators: epidemiology in France 2002-2003.

J Periodontal Res. 2006 Aug;41(4):253-8.

[4] Borrell L.N. et al. Prevalence and trends in periodontitis in the USA: from the NHANES III to the NHANES, 1988 to 2000. J Dent Res (2005) 84 :924-930.

[5] Chapple I.L.C. et al. Diabetes and periodontal diseases: consensus report of the Joint EFP/AAP Workshop on Periodontitis and Systemic Diseases. J Periodontol (2013) 84:S106-S112.

[6] Borgnakke W.S. et al. Effect of periodontal disease on diabetes: systematic review of epidemiologic observational evidence. J Clin Periodontol (2013) 40 :S135-S152.

[7] Taylor J.J., Preshaw P.M., Lalla E. A review of the evidence for pathogenic mechanisms that may link periodontitis and diabetes. J Clin Periodontol (2013) 40:S113-S134.

[8] Engebretson, S. et al. The influence of diabetes on gingival crevicular fluid betaglucuronidase and interleukin-8.

J Clin Periodontology (2006) 11: 784-790.
[9] Salvi G.E. et al. Inflammatory mediator response as a potential risk marker for periodontal diseases in insulin-dependent diabetes mellitus patients. J Periodontol (1997) 68:127-135

[10] Duarte P.M. Role of smoking and type 2 diabetes in the immunobalance of advanced chronic periodontitis. J Periodontology (2011) 82:429-438

[11] Takano et al. The effect of systemic antitumor necrosis factor-alpha treatment on Porphyromonas gingivalis infection in type 2 diabetic mice. Archives of Oral Biology (2010) 55:79-384.

[12] Vieira Ribeiro et al. Cytokines and bonerelated factors in systemically healthy patients with chronic periodontitis and patients with type 2 diabetes and chronic periodontitis. J Periodontology (2008) 79:1208-1216.

[13] Rangé H. et al. Risque Parodontal chez le patient obèse. Obesité (2010) $5: 67-73$.

[14] Schmidt et al. Advanced glycation endproducts (AGEs) induce oxidant stress in the gingiva: a potentiam mechanism underlying accelerated periodontal disease associated with diabetes. J Periodontal Research (1996) 31:508-515.

[15] Takeda et al. Relationship of serum advanced glycation end products with deterioration of periodontitis in type 2 diabetes patients. J Periodontology (2006) 77: 15-20.

[16] Lalla et al. Blockade of RAGE suppresses periodontitis associated bone loss in diabetic mice. J Clin Invest (2000) 105: 1117-1124.

[17] Makiura et al. Relationship of Porphyromonas gingivalis with glycemic level in patients with type 2 diabetes following periodontal treatment. Oral Microbiology and Immunol (2008) 23:348-351.

[18] D'Aiuto et al. Oxidative stress, systemic inflammation, and severe periodontitis. J Dent Res (2010) 89:1241-1246.

[19] Engebretson, S. et al. Evidence that periodontal treatment improves diabetes outcomes: a systematic review and metaanalysis. J Periodontol (2013) 84 :S153-S163.

[20] Teeuw W.J. et al. Effect of Periodontal Treatment on Glycemic Control of Diabetic Patients: A systematic review and metaanalysis. Diabetes Care (2010) 33 :421-427.

[21] Simpson T.C. et al. Treatment of periodontal disease for glycaemic control in people with diabetes. Cochrane Database Syst $\operatorname{Rev}(2010) 12$ : CD004714

[22] Darré, L. et al. Efficacy of periodontal treatment on glycaemic control in diabetic patients: A meta-analysis of interventional studies. Diabetes Metab (2008) 34 :497-506.

[23] Martorelli de Lima A.F. et al. Therapy with adjunctive doxycycline local delivery in patients with type 1 diabetes mellitus and periodontitis. J Clin Periodontol (2004) 31 :648-653.

[24] Llambes F. et al. Effect of non-surgical periodontal treatment with or without doxycycline on the periodontium of type 1 diabetic patients. J Clin Periodontol (2005) 32 :915-920.

[25] Christgau M. et al. Healing response to non-surgical periodontal therapy in patients with diabetes mellitus: clinical, microbiological, and immunologic results. J Clin Periodontol (1998) 25:112-124. 